\title{
Characteristics and self-rated health of overlap syndrome
}

This article was published in the following Dove Press journal:

International Journal of COPD

21 July 2014

Number of times this article has been viewed

\author{
Jung Wha Chung' \\ Kyoung Ae Kong ${ }^{2}$ \\ Jin Hwa Lee' \\ Seok Jeong Lee' \\ Yon Ju Ryu' \\ Jung Hyun Chang'
}

'Division of Pulmonary and Critical Care Medicine, Department of Internal Medicine, School of Medicine, Ewha Womans University, ${ }^{2}$ Clinical Trial Center, Ewha Womans University Mokdong Hospital, Seoul, Korea
Correspondence: Jin Hwa Lee Division of Pulmonary and Critical Care Medicine, Department of Internal Medicine, School of Medicine, Ewha Womans University, I07I Anyangcheon-ro, Yangcheon-gu, Seoul I58-710, Korea

Email jinhwalee@ewha.ac.kr
Background and objective: Overlap syndrome shares features of both asthma and chronic obstructive pulmonary disease (COPD). The aim of this study was to investigate characteristics of overlap syndrome and their effect on self-rated health (SRH).

Methods: We analyzed data from the Fourth Korea National Health and Nutrition Examination Survey of 2007-2009. Subjects with acceptable spirometry and available wheezing history were included. Subjects were classified into four groups based on forced expiratory volume in one second $\left(\mathrm{FEV}_{1}\right)$ /forced vital capacity $(\mathrm{FVC})$ results and the presence or absence of selfreported wheezing for the previous 12 months: 1) COPD group, defined as having FEV $/ \mathrm{FVC}$ $<0.7$ without self-reported wheezing history; 2) asthma group, defined as having self-reported wheezing history without $\mathrm{FEV}_{1} / \mathrm{FVC}<0.7 ; 3$ ) overlap syndrome group, having both $\mathrm{FEV}_{1} /$ FVC $<0.7$ and wheezing history; and 4) non-obstructive disease (NOD) group, having neither $\mathrm{FEV}_{1} / \mathrm{FVC}<0.7$ nor self-reported wheezing. SRH was categorized as better or lower based on responses to a questionnaire.

Results: From a total 9,104 subjects, 700 were assigned to the COPD group, 560 to the asthma group, 210 to the overlap syndrome group, and 7,634 to the NOD group. Compared to the other groups, subjects in the overlap syndrome group were more likely to have low lung function, a high proportion of smokers, low socioeconomic status, short education duration, lower SRH, and past diagnosis of pulmonary tuberculosis or bronchiectasis. Multiple logistic regression analysis revealed that both overlap syndrome and asthma groups were independently associated with lower SRH after adjustment for age, sex, socioeconomic status, education level, smoking status, comorbidities, and lung function. Female, old age, low education level, low economic status, smoker and other comorbidities were also associated with lower SRH.

Conclusion: Overlap syndrome was accompanied by high morbidity and was associated with lower SRH, which needs more appropriate care.

Keywords: chronic obstructive lung disease, asthma, overlap syndrome, self-rated health, COPD

\section{Introduction}

Chronic obstructive pulmonary disease (COPD) and asthma are the most common airway diseases. While COPD patients are likely to be older, smokers, and have persistent and usually progressive airflow limitation, patients with asthma tend to be younger, nonsmokers, and have atopy and/or allergies and reversible airway obstruction. ${ }^{1}$ However, in practice, airflow limitation that is not fully reversible is insufficient to explain COPD. Since some patients with asthma also develop poorly reversible airway limitation, differential diagnosis is sometimes difficult for clinicians. In the elderly with COPD, variable airway obstruction is commonly observed. Approximately half of COPD patients have 
both COPD and asthma (overlap syndrome), and coexistence of the two diseases increases with age. ${ }^{2}$ Increased airway hyper-responsiveness (AHR) is reported to be associated with increased COPD exacerbations and overall mortality. ${ }^{3}$

Overlap syndrome is defined as having both AHR and incompletely reversible airway obstruction. ${ }^{4,5}$ In patients with overlap syndrome, forced expiratory volume in one second $\left(\mathrm{FEV}_{1}\right)$ does not return to normal and often worsens over time. Since these patients are generally excluded from studies examining either disease, the clinical implications of overlap syndrome remain poorly recognized. ${ }^{4}$ Despite a high prevalence of COPD and asthma in the general Korean population, ${ }^{6-8}$ clinical features of patients with overlap syndrome have not been well described.

The aim of our study was to characterize overlap syndrome and investigate its impact on self-rated health (SRH) by using the data from the Fourth Korea National Health and Nutrition Examination Survey of 2007-2009 (KNHANES IV survey). ${ }^{8}$

\section{Methods}

\section{Subjects}

Data from the KNHANES IV survey conducted between July 2007 and December 2009 were reviewed retrospectively. The survey was conducted as a cross-sectional observational study using complex, stratified multistage cluster sampling. It was designed as a rolling sample survey based on the 2005 Korean population and housing census; once subjects were interviewed, they were not interviewed again. Data were obtained from independent samples for each year, representing the total non-institutionalized population of Korea. Among the 24,971 potential subjects, 18,406 were $>19$ years. We excluded subjects with unavailable respiratory symptoms and poor spirometry results; that is, results that did not meet an acceptable flow-volume loop or were not reproducible. Finally, 9,104 subjects were enrolled (Figure 1). The study was approved by the Korea Centers for Disease Control and Prevention Institutional Review Board, and informed consent was obtained from each participant.

Medical histories of respiratory symptoms and comorbidities, duration of education, and socioeconomic status were obtained by questionnaire. Height, weight, waist circumference, and blood pressure were measured in all subjects. Triglyceride and high-density lipoprotein (HDL) cholesterol levels were obtained after 12 hours of fasting. Spirometry was performed on all subjects $>19$ years. FEV and forced vital capacity (FVC) were measured, and a predicted ratio for each parameter was calculated based on age, sex, height, and race.

\section{Definitions}

Subjects were assigned to one of four groups based on data reported for the previous 12 months: 1) the COPD group was defined as subjects having an $\mathrm{FEV}_{1} / \mathrm{FVC}$ ratio $<0.7$ without history of self-reported wheezing; 2) the asthma

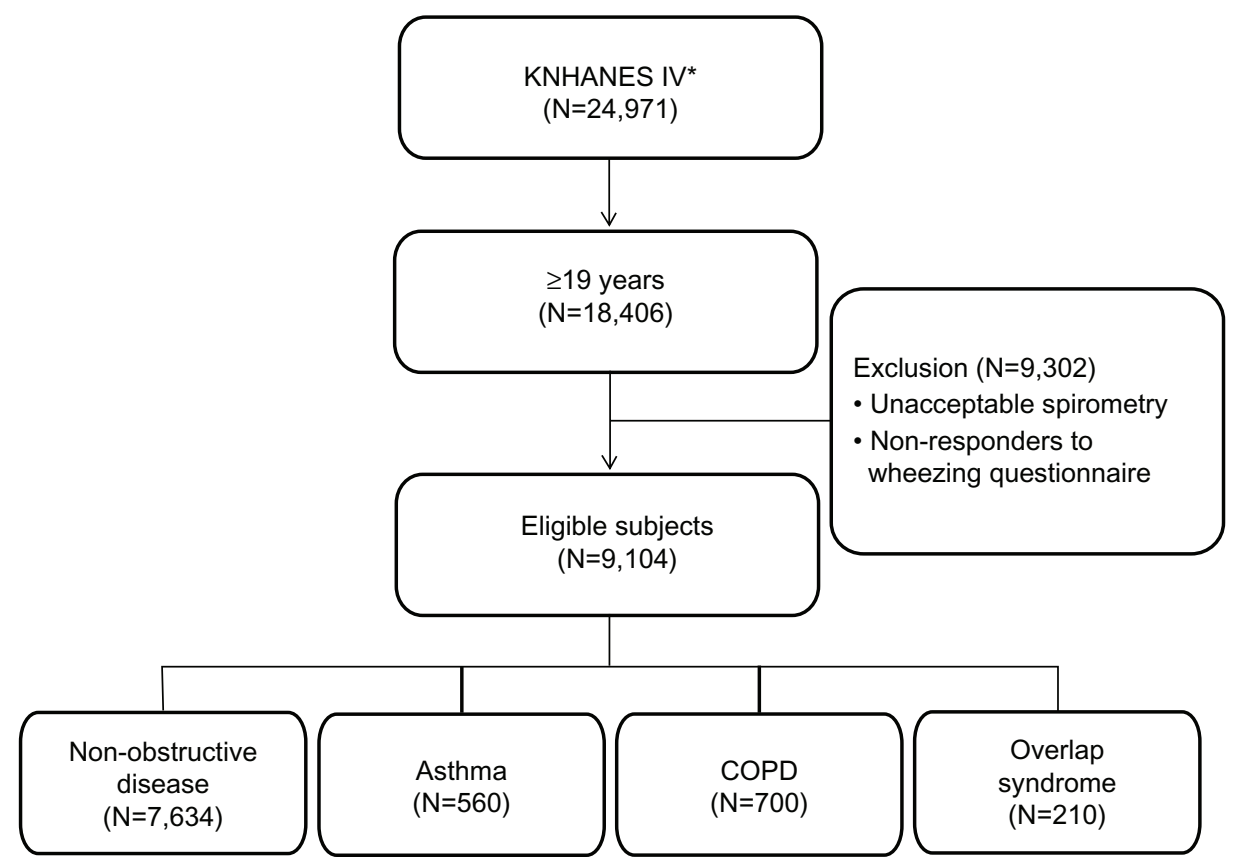

Figure I Study design.

Note: *KNHANES IV, Fourth Korea National Health and Nutrition Examination Survey, 2007-2009. ${ }^{8}$

Abbreviation: COPD, chronic obstructive pulmonary disese. 
group was comprised of subjects with a history of wheezing without $\mathrm{FEV}_{1} / \mathrm{FVC}<0.7 ; 3$ ) the overlap syndrome group included subjects with both $\mathrm{FEV}_{1} / \mathrm{FVC}<0.7$ and a history of self-reported wheezing; the remaining subjects made up the non-obstructive disease (NOD) group.

SRH was assessed by asking the following question: "Would you say your general health status is excellent, very good, good, fair, or poor?" Based on responses, subjects were categorized into two outcome measures: better (excellent, very good, and good), and lower (fair and poor). ${ }^{9}$ Standard questions were used to assess underlying disease such as, "Has a medical doctor ever told you that you had the following diseases: COPD, asthma, pulmonary tuberculosis, bronchiectasis, hypertension, diabetes mellitus, cerebrovascular disease, osteoarthritis, allergic rhinitis, or atopic dermatitis?" with the response alternatives "Yes" or "No" for each option. They were also asked about their wheezing history using the question: "Have you had wheezing or whistling in your chest at any time during the previous year?" Metabolic syndrome was diagnosed when more than three of the five criteria outlined by the National Cholesterol Education Program's Adult Treatment Panel III (NCEP/ATP III) were met. ${ }^{10}$ Mean blood pressure was used for diagnosis of metabolic syndrome. Never-smoker was defined as having smoked less than five packs of cigarettes during their lifetime.

\section{Statistical analyses}

Descriptive analysis was performed for all variables. Categorical variables were analyzed by chi-square test. Regression analysis for sampling survey data and Tukey's post hoc test were used to compare continuous numeric variables among the four groups. All estimates were calculated using the appropriate sampling weight provided in KNHANES IV, and standard errors (SE) were calculated reflecting complex design and survey weight. Simple logistic regression analysis was used to evaluate association between individual factors and lower SRH, and multiple logistic regression analysis of possible risk factors was performed using SAS version 9.2 statistical software (SAS institute Inc., Cary NC, USA). A $P$-value $<0.05$ was considered statistically significant.

\section{Results}

\section{Subject characteristics}

Of the 9,104 subjects with eligible spirometry and available wheezing history, 700 were admitted to the COPD group; 560 to the asthma group; 210 to the overlap syndrome group; and 7,634 to the NOD group. Epidemiological characteristics of the subjects are summarized in Table 1 and comparisons between included and excluded subjects are shown in Table S1.

Table 1 shows that the mean age of all subjects was 44.4 years, whereas mean age of the COPD group was 60.7 years, and that of the overlap syndrome group 60.4 years. The overlap syndrome and COPD groups showed a higher proportion of males compared to the asthma group (67\% and $69 \%$, respectively, versus $46 \%$ ). There were more smokers in the overlap syndrome group (73\%) than in the COPD, asthma, and NOD groups (65\%, 54\%, and 44\%, respectively). Subjects with overlap syndrome had the highest proportion of low socioeconomic status and fewer than 6 years of education, followed by the COPD and asthma groups. Metabolic syndrome and osteoarthritis were most prevalent in the overlap syndrome group. Except for metabolic syndrome, all comorbidities were more common in the COPD and overlap syndrome groups than the asthma group, which was likely due to their higher mean age. Despite higher mean age and prevalence of comorbidities (except for metabolic syndrome), the COPD group showed better SRH than the asthma group.

To identify factors associated with each airway disease, we performed multinomial logistic regression analysis; results are summarized in Table 2 . While sex was associated with both asthma and COPD, old age increased the risks in both COPD and overlap syndrome groups. Low socioeconomic status was associated with overlap syndrome, and short education duration and smoking status were associated with all airway diseases. While lower body mass index (BMI) increased risk of both COPD and overlap syndrome, being overweight seemed to decrease risk of COPD. Except for osteoarthritis, other comorbidities were not associated with AHR.

Table 3 shows the lung function of subjects. $\mathrm{FEV}_{1} / \mathrm{FVC}$, $\mathrm{FEV}_{1}$, and $\mathrm{FVC}$ were lowest in the overlap syndrome group. Table 4 shows the history of respiratory and allergic disease based on questionnaire responses. Subjects previously diagnosed with pulmonary tuberculosis or bronchiectasis were the most common subjects in the overlap syndrome group compared to the other three groups.

\section{Factors associated with lower SRH}

Independent factors associated with lower SRH are summarized in Table 5. In a multiple logistic regression analysis using NOD as a reference, overlap syndrome was the strongest factor associated with lower SRH (odds ratio [OR]: 2.31, 95\% confidence interval [CI]: $1.51-3.55, P<0.001)$, followed by the asthma group (OR: 2.04, 95\% CI: 1.58-2.65, $P<0.001$ ). 
Table I Epidemiological characteristics

\begin{tabular}{|c|c|c|c|c|c|c|}
\hline Variables & $\begin{array}{l}\text { Overall } \\
(N=9,104)\end{array}$ & $\begin{array}{l}\text { NOD } \\
(N=7,634)\end{array}$ & $\begin{array}{l}\text { Asthma } \\
(\mathrm{N}=560)\end{array}$ & $\begin{array}{l}\text { COPD } \\
(\mathrm{N}=700)\end{array}$ & $\begin{array}{l}\text { Overlap syndrome } \\
(\mathrm{N}=2 \mid 0)\end{array}$ & $P$-value \\
\hline \multicolumn{7}{|l|}{$\operatorname{Sex}(\%)$} \\
\hline Male & 4,067 & $3,216(49)$ & $221(46)$ & $500(69)$ & $130(67)$ & $<0.001$ \\
\hline \multicolumn{7}{|l|}{ Age, years } \\
\hline Mean \pm SE & $44.4 \pm 0.3$ & $42.9 \pm 0.3$ & $44.8 \pm 0.9$ & $60.7 \pm 0.9$ & $60.4 \pm 1.9$ & $<0.001 *$ \\
\hline $19-39$ & 2,410 & $2,223(45)$ & $150(44)$ & $28(10)$ & $9(11)$ & $<0.001$ \\
\hline $40-59$ & 4,062 & $3,593(40)$ & $216(34)$ & $184(30)$ & $69(34)$ & \\
\hline$\geq 60$ & 2,632 & $1,818(15)$ & $194(22)$ & $488(60)$ & $132(55)$ & \\
\hline \multicolumn{7}{|l|}{ Socioeconomic status } \\
\hline$>75$ th percentile & 2,354 & I,999 (26) & I5I (29) & $163(23)$ & $41(15)$ & 0.025 \\
\hline $25-75$ th percentile & 4,597 & $3,893(5 \mathrm{I})$ & $256(47)$ & $35 I(52)$ & $97(50)$ & \\
\hline$<25$ th percentile & 2,153 & $\mathrm{I}, 742(23)$ & $153(24)$ & $186(25)$ & $72(35)$ & \\
\hline \multicolumn{7}{|l|}{ Education duration, years } \\
\hline$>12$ & 2,270 & $2,048(3 I)$ & $116(26)$ & $90(14)$ & $16(8)$ & $<0.001$ \\
\hline $9-12$ & 3,070 & $2,726(42)$ & $167(39)$ & $140(23)$ & $37(20)$ & \\
\hline $6-9$ & 1,196 & $972(10)$ & $64(10)$ & $127(19)$ & $33(16)$ & \\
\hline$\leq 6$ & 2,568 & I,888 (I7) & $213(25)$ & $343(44)$ & $106(54)$ & \\
\hline \multicolumn{7}{|l|}{ Self-rated health } \\
\hline Better & 6,957 & $6,010(83)$ & $334(67)$ & $507(76)$ & $113(53)$ & $<0.00 \mathrm{I}$ \\
\hline Lower & 2,147 & $\mathrm{I}, 624(17)$ & $226(33)$ & $193(24)$ & $104(46)$ & \\
\hline \multicolumn{7}{|l|}{ Smoking history } \\
\hline Never-smoker & 5,327 & $4,728(57)$ & $298(46)$ & $230(35)$ & $71(28)$ & $<0.00 \mathrm{I}$ \\
\hline Ex-smoker & 1,696 & I,348 (18) & $44(6)$ & $252(33)$ & $52(28)$ & \\
\hline Current smoker & $2,08 I$ & I,558 (26) & $218(48)$ & $218(32)$ & $87(45)$ & \\
\hline \multicolumn{7}{|l|}{$\mathrm{BMI}, \mathrm{kg} / \mathrm{m}^{2}$} \\
\hline Mean \pm SE & $24.2 \pm 0.1$ & $24.3 \pm 0.1$ & $24.8 \pm 0.2$ & $23.3 \pm 0.2$ & $23.9 \pm 0.4$ & $<0.00 \mathrm{I}$ \\
\hline$<18.5$ & 202 & $154(2)$ & $7(2)$ & $28(5)$ & $13(5)$ & $<0.001$ \\
\hline $18.5-25$ & 5,518 & $4,604(60)$ & $282(53)$ & $499(68)$ & $133(63)$ & \\
\hline$>25$ & 3,384 & $2,876(38)$ & $27 \mid(45)$ & $173(28)$ & $64(3 I)$ & \\
\hline \multicolumn{7}{|l|}{ Waist circumference, $\mathrm{cm}$} \\
\hline Mean \pm SE & $82.7 \pm 0.2$ & $82.6 \pm 0.2$ & $84.5 \pm 0.7$ & $82.9 \pm 0.5$ & $84.9 \pm 1.0$ & $>0.050 * *$ \\
\hline \multicolumn{7}{|l|}{ Comorbidities } \\
\hline Hypertension & $\mathrm{I}, 892$ & I,446 (I5) & $158(19)$ & $217(29)$ & 7I (28) & $<0.00$ I \\
\hline Diabetes mellitus & 716 & $550(6)$ & $51(6)$ & $91(13)$ & $24(13)$ & $<0.001$ \\
\hline Cerebrovascular disease & 342 & $244(2)$ & $30(4)$ & $55(6)$ & $13(6)$ & $<0.00 \mathrm{I}$ \\
\hline Metabolic syndrome*** & 1,639 & $\mathrm{I}, 3 \mathrm{I} 2(25)$ & $148(34)$ & $128(32)$ & $51(39)$ & $<0.001$ \\
\hline Osteoarthritis & $|, 35|$ & $1,028(10)$ & $144(17)$ & $132(18)$ & $47(23)$ & $<0.001$ \\
\hline
\end{tabular}

Notes: Data are presented as mean \pm SE or number (percentage). $* P<0.00$ I were obtained except asthma versus NOD group $(P=0.173)$ and $C O P D$ versus overlap syndrome group $(P=0.998)$ by post hoc Tukey's method; $* * P>0.05$ were obtained except asthma group versus NOD group, $P=0.02$ I by post hoc Tukey's method. $* * * A$ total of 5,55 I subjects were analyzed.

Abbreviations: NOD, non-obstructive disease; COPD, chronic obstructive pulmonary disease; N, number of subjects; BMI, body mass index; SE, standard error.

Adjusting for other factors such as sex, age, smoking, other comorbidities and $\mathrm{FEV}_{1}$, the COPD group lost statistical significance. In addition, female sex, age $>60$ years, low socioeconomic status, education duration $<9$ years, presence of smoking history, and $\mathrm{FEV}_{1}<50 \%$ were also associated with lower SRH. Neither obesity (BMI $\geq 25 \mathrm{~kg} / \mathrm{m}^{2}$ ) nor low weight (BMI $<18.5 \mathrm{~kg} / \mathrm{m}^{2}$ ) were associated with lower SRH.

\section{Discussion}

The current study demonstrated that the overlap syndrome group makes up a significant portion of obstructive lung disease with high burden of morbidity based on data from a national population-based survey. Overlap syndrome was also independently associated with lower SRH. Subjects were classified into four groups using $\mathrm{FEV}_{1} / \mathrm{FVC}$ results for airway obstruction and wheezing history as markers of AHR. These groups showed significant differences in variable individual factors during analyses, which provided validity to the study design. Each group represented a unique disease entity. For example, the COPD group had a greater number of clinical factors (increased age, higher prevalence of smoking, and comorbidities) compared to the asthma group. The overlap syndrome group showed a similar trend 
Table 2 Multinomial logistic regression analysis to identify factors associated with airway diseases

\begin{tabular}{|c|c|c|c|c|c|c|c|c|c|}
\hline \multirow[t]{2}{*}{ Variables } & \multicolumn{3}{|c|}{ Asthma } & \multicolumn{3}{|c|}{ COPD } & \multicolumn{3}{|c|}{ Overlap syndrome } \\
\hline & OR & $95 \% \mathrm{Cl}$ & $P$-value & OR & $95 \% \mathrm{Cl}$ & $P$-value & OR & $95 \% \mathrm{Cl}$ & $P$-value \\
\hline \multicolumn{10}{|l|}{ Sex } \\
\hline Male & 1 & & & 1 & & & 1 & & \\
\hline Female & 1.81 & $1.28-2.58$ & 0.0009 & 0.41 & $0.28-0.60$ & $<0.0001$ & 0.61 & $0.34-1.07$ & 0.087 \\
\hline \multicolumn{10}{|l|}{ Age, years } \\
\hline $19-39$ & 1 & & & 1 & & & 1 & & \\
\hline $40-59$ & 0.88 & $0.66-1.17$ & NS & 2.90 & I.82-4.63 & $<0.0001$ & 2.77 & I.09-7.04 & 0.032 \\
\hline$\geq 60$ & 1.26 & $0.84-1.89$ & NS & 14.53 & $8.83-23.90$ & $<0.0001$ & 9.50 & $3.39-26.63$ & $<0.000$ I \\
\hline \multicolumn{10}{|c|}{ Socioeconomic status, percentile } \\
\hline$>75$ th & 1 & & & 1 & & & I & & \\
\hline$<25$ th & 0.79 & $0.58-1.08$ & NS & 1.12 & $0.83-1.50$ & NS & 1.87 & $1.11-3.16$ & 0.02 \\
\hline $25-75$ th & 0.75 & $0.57-0.99$ & 0.04 & 1.07 & $0.8 \mathrm{I}-1.4 \mathrm{I}$ & NS & 1.42 & $0.90-2.25$ & NS \\
\hline \multicolumn{10}{|c|}{ Education duration, years } \\
\hline$>12$ & 1 & & & 1 & & & I & & \\
\hline$\leq 6$ & 1.45 & $1.00-2.09$ & 0.048 & 2.16 & $1.46-3.20$ & 0.0001 & 4.28 & I.87-9.79 & 0.0006 \\
\hline $6-9$ & 1.15 & $0.75-1.76$ & NS & 2.08 & $1.38-3.13$ & 0.0004 & 2.58 & $1.00-6.62$ & 0.049 \\
\hline $9-12$ & 1.03 & $0.75-1.42$ & NS & 1.13 & $0.78-1.64$ & NS & 1.32 & $0.65-2.68$ & NS \\
\hline \multicolumn{10}{|l|}{ Smoking } \\
\hline Never-smoker & I & & & I & & & 1 & & \\
\hline Ex-smoker & 0.67 & $0.42-1.06$ & 0.085 & 1.50 & $1.04-2.17$ & 0.032 & 2.64 & $1.44-4.83$ & 0.002 \\
\hline Current & 4.06 & $2.92-5.64$ & $<0.0001$ & 1.99 & I.39-2.87 & 0.0002 & 5.07 & $3.04-8.46$ & $<0.000$ I \\
\hline \multicolumn{10}{|l|}{$\mathrm{BMI}, \mathrm{kg} / \mathrm{m}^{2}$} \\
\hline $18.5-25$ & 1 & & & I & & & 1 & & \\
\hline$<18.5$ & 0.85 & $0.32-2.26$ & NS & 2.14 & $1.05-4.35$ & 0.036 & 2.57 & I.18-5.57 & 0.02 \\
\hline$\geq 25$ & 1.25 & $0.99-1.58$ & 0.056 & 0.65 & $0.5 \mathrm{I}-0.83$ & 0.0005 & 0.74 & $0.48-1.14$ & NS \\
\hline \multicolumn{10}{|l|}{ Hypertension } \\
\hline No & I & & & I & & & 1 & & \\
\hline Yes & 1.02 & $0.77-1.35$ & NS & 0.86 & $0.68-1.08$ & NS & 0.86 & $0.57-1.30$ & NS \\
\hline \multicolumn{10}{|l|}{ Diabetes mellitus } \\
\hline No & 1 & & & $\mathrm{I}$ & & & 1 & & \\
\hline Yes & 0.86 & $0.57-1.31$ & NS & 1.05 & $0.77-1.44$ & NS & 1.12 & $0.62-2.03$ & NS \\
\hline \multicolumn{10}{|c|}{ Cerebrovascular disease } \\
\hline No & 1 & & & I & & & 1 & & \\
\hline Yes & 1.56 & $0.97-2.53$ & 0.068 & 0.87 & $0.60-1.28$ & NS & 0.92 & $0.43-1.95$ & NS \\
\hline \multicolumn{10}{|l|}{ Osteoarthritis } \\
\hline No & 1 & & & I & & & 1 & & \\
\hline Yes & 1.61 & $1.19-2.17$ & 0.002 & 1.09 & $0.78-1.52$ & NS & $\mathrm{I} .4 \mathrm{I}$ & $0.87-2.31$ & NS \\
\hline
\end{tabular}

Abbreviations: COPD, chronic obstructive pulmonary disease; OR, odds ratio; $\mathrm{Cl}$, confidence interval; NS, not significant; BMI, body mass index.

Table 3 Lung function

\begin{tabular}{|c|c|c|c|c|c|c|}
\hline Variables & $\begin{array}{l}\text { Overall } \\
(N=9,104)\end{array}$ & $\begin{array}{l}\text { NOD } \\
(\mathrm{N}=7,634)\end{array}$ & $\begin{array}{l}\text { Asthma } \\
(\mathrm{N}=560)\end{array}$ & $\begin{array}{l}\text { COPD } \\
(\mathrm{N}=700)\end{array}$ & $\begin{array}{l}\text { Overlap syndrome } \\
(\mathrm{N}=2 \mid 0)\end{array}$ & $P$-value \\
\hline \multicolumn{7}{|l|}{ Lung function } \\
\hline $\mathrm{FEV}_{1} / \mathrm{FVC}$ & $0.80 \pm 0.0$ & $0.82 \pm 0.0$ & $0.8 \mathrm{I} \pm 0.0$ & $0.64 \pm 0.0$ & $0.60 \pm 0.0$ & $<0.00 I^{* *}$ \\
\hline $\mathrm{FEV}, \%$ predicted & $92.0 \pm 0.2$ & $93.6 \pm 0.2$ & $90.4 \pm 0.7$ & $77.2 \pm 0.7$ & $69.4 \pm 1.5$ & $<0.001$ \\
\hline FVC\% predicted & $92.9 \pm 0.2$ & $93.3 \pm 0.2$ & $90.9 \pm 0.7$ & $90.0 \pm 0.7$ & $86.2 \pm 1.4$ & $<0.050^{\ddagger}$ \\
\hline \multicolumn{7}{|l|}{$\mathrm{FEV}_{1} \%$ predicted } \\
\hline$\geq 80$ & 7,616 & 6,796 (89) & $448(8 I)$ & $320(45)$ & $52(27)$ & $<0.001$ \\
\hline $50-80$ & I,422 & 835 (II) & $110(19)$ & $35 \mathrm{I}(5 \mathrm{I})$ & $126(6 \mathrm{I})$ & \\
\hline$<50$ & 66 & $3(<1)$ & $2(<I)$ & $29(4)$ & $32(12)$ & \\
\hline
\end{tabular}

Notes: Data are presented as mean \pm SE or number (percentage). ${ }^{* *} P<0.00$ I were obtained except asthma versus NOD group, $P=0.004$ by post hoc Tukey's method. $\ddagger P<0.050$ were obtained except COPD group versus asthma group $(P=0.794)$, COPD group versus overlap syndrome group $(P=0.060)$ by post hoc Tukey's method.

Abbreviations: NOD, non-obstructive disease; COPD, chronic obstructive pulmonary disease; $\mathrm{N}$, the number of subjects; $\mathrm{FEV}$, forced expiratory volume in one second; FVC, forced volume capacity; SE, standard error. 
Table 4 Past history of respiratory or allergic diseases based on responses to questionnaires*

\begin{tabular}{|c|c|c|c|c|c|}
\hline Diseases & $\begin{array}{l}\text { Overall } \\
(N=9,104)\end{array}$ & $\begin{array}{l}\text { NOD } \\
(\mathrm{N}=7,634)\end{array}$ & $\begin{array}{l}\text { Asthma } \\
(\mathrm{N}=560)\end{array}$ & $\begin{array}{l}\text { COPD } \\
(\mathrm{N}=700)\end{array}$ & $\begin{array}{l}\text { Overlap syndrome } \\
(\mathrm{N}=2 \mid 0)\end{array}$ \\
\hline COPD & $64(1)$ & $28(<\mathrm{I})$ & $9(2)$ & II (I) & $16(7)$ \\
\hline Asthma & $325(3)$ & $116(1)$ & $95(14)$ & $34(6)$ & $80(40)$ \\
\hline COPD and asthma & $15(<1)$ & $\mathrm{I}(<\mathrm{I})$ & $3(1)$ & $\mathrm{I}(<\mathrm{I})$ & $10(4)$ \\
\hline Tuberculosis & $533(5)$ & $365(4)$ & $31(7)$ & $94(15)$ & $43(21)$ \\
\hline Bronchiectasis & $57(\mathrm{I})$ & $23(<1)$ & $7(1)$ & $10(1)$ & $17(6)$ \\
\hline Allergic rhinitis & $862(\mathrm{II})$ & $726(\mathrm{II})$ & $75(15)$ & $4 \mid(7)$ & $20(10)$ \\
\hline Atopic dermatitis & $216(3)$ & $180(3)$ & $24(7)$ & $8(1)$ & $4(I)$ \\
\hline
\end{tabular}

Notes: Data are presented as number (percentage). *In all comparisons, $P<0.00 \mathrm{I}$ by chi-square test.

Abbreviations: NOD, non-obstructive disease; COPD, chronic obstructive pulmonary disease; N, number of subjects.

Table 5 Factors associated with lower self-rated health $(\mathrm{N}=9,104)$

\begin{tabular}{|c|c|c|c|c|c|c|}
\hline \multirow[t]{2}{*}{ Variables } & \multicolumn{3}{|c|}{ Univariate analysis } & \multicolumn{3}{|c|}{ Multivariate analysis } \\
\hline & OR & $95 \% \mathrm{Cl}$ & $P$-value & OR & $95 \% \mathrm{Cl}$ & $P$-value \\
\hline \multicolumn{7}{|l|}{ Sex } \\
\hline Male & 1.00 & & & 1.00 & & \\
\hline Female & 2.04 & $1.78-2.34$ & $<0.001$ & 2.30 & $1.83-2.90$ & $<0.001$ \\
\hline \multicolumn{7}{|l|}{ Age, years } \\
\hline $19-39$ & 1.00 & & & 1.00 & & \\
\hline $40-59$ & 1.71 & $1.45-2.01$ & $<0.001$ & 1.16 & $0.97-1.39$ & 0.105 \\
\hline$\geq 60$ & 3.99 & $3.32-4.79$ & $<0.001$ & 1.29 & $1.00-1.66$ & 0.049 \\
\hline \multicolumn{7}{|l|}{ Socioeconomic status } \\
\hline$>75$ th percentile & 1.00 & & & 1.00 & & \\
\hline 25-75th percentile & 1.32 & $1.12-1.57$ & 0.001 & 1.23 & $1.02-1.48$ & 0.028 \\
\hline$<25$ th percentile & 1.69 & I.39-2.04 & $<0.001$ & 1.43 & $1.16-1.76$ & 0.001 \\
\hline \multicolumn{7}{|l|}{ Educational duration, years } \\
\hline$>12$ & 1.00 & & & 1.00 & & \\
\hline $9-12$ & 1.39 & $1.15-1.67$ & 0.005 & 1.15 & $0.95-1.39$ & 0.145 \\
\hline $6-9$ & 2.26 & I.79-2.85 & $<0.001$ & 1.35 & $1.05-1.74$ & 0.018 \\
\hline$\leq 6$ & 4.77 & $3.96-5.74$ & $<0.001$ & 1.91 & $1.52-2.40$ & $<0.001$ \\
\hline \multicolumn{7}{|l|}{ Smoking history } \\
\hline Non-smoker & 1.00 & & & 1.00 & & \\
\hline Ex-smoker & 0.84 & $0.70-1.00$ & 0.055 & 1.42 & I.09-I.84 & 0.009 \\
\hline Current smoker & 0.70 & $0.60-0.82$ & $<0.001$ & 1.36 & $1.06-1.75$ & 0.016 \\
\hline \multicolumn{7}{|l|}{ Airway disease } \\
\hline NOD & 1.00 & & & 1.00 & & \\
\hline COPD & 1.51 & $1.20-1.89$ & 0.001 & 0.92 & $0.69-1.22$ & 0.559 \\
\hline Asthma & 2.33 & $1.84-2.95$ & $<0.001$ & 2.04 & $1.58-2.65$ & $<0.001$ \\
\hline Overlap syndrome & 4.14 & $2.82-6.08$ & $<0.001$ & 2.31 & $1.5 \mathrm{I}-3.55$ & $<0.001$ \\
\hline \multicolumn{7}{|l|}{ Comorbidities } \\
\hline Hypertension & 2.93 & $2.53-3.40$ & $<0.001$ & 1.53 & $1.29-1.83$ & $<0.001$ \\
\hline Diabetes mellitus & 3.78 & $3.08-4.64$ & $<0.001$ & 2.33 & $1.83-2.97$ & $<0.001$ \\
\hline Cerebrovascular disease & 5.64 & $4.33-7.34$ & $<0.001$ & 3.10 & $2.23-4.31$ & $<0.001$ \\
\hline Osteoarthritis & 3.84 & $3.30-4.48$ & $<0.001$ & 1.86 & $1.57-2.19$ & $<0.001$ \\
\hline \multicolumn{7}{|l|}{ BMI, kg/m² } \\
\hline $18.5-25$ & 1.00 & & & 1.00 & & \\
\hline$<18.5$ & 0.97 & $0.63-1.48$ & 0.877 & 0.97 & $0.62-|.5|$ & 0.895 \\
\hline$\geq 25$ & 1.25 & $1.09-1.45$ & 0.002 & 1.12 & $0.95-1.32$ & 0.161 \\
\hline \multicolumn{7}{|l|}{$\mathrm{FEV}_{1} \%$ predicted } \\
\hline$\geq 80$ & 1.00 & & & 1.00 & & \\
\hline $50-80$ & 1.45 & $|.23-1.7|$ & $<0.001$ & 1.16 & $0.96-|.4|$ & 0.120 \\
\hline$<50$ & 4.91 & $2.69-8.96$ & $<0.001$ & 2.82 & $1.34-5.90$ & 0.006 \\
\hline
\end{tabular}

Abbreviations: $\mathrm{OR}$, odds ratio; $\mathrm{Cl}$, confidence interval; NOD, non-obstructive disease; COPD, chronic obstructive pulmonary disease; $\mathrm{BMI}$, body mass index; $\mathrm{FEV}{ }_{\text {, }}$, forced expiratory volume in one second; $\mathrm{N}$, number of subjects. 
as the COPD group, but most of the clinical factors were worse than those of the COPD group, except for comorbidities. Among subjects of overlap syndrome, almost half of them presented lower SRH, which is the highest rate among the four groups. These results were very similar to those from a previous study that demonstrated that adults with overlap syndrome, asthma, or COPD reported more health impairments compared to those with neither disease, and adults with overlap syndrome and COPD were more likely to have comorbidities. ${ }^{11}$

Despite these distinctive clinical characteristics, the physician diagnostic rate for obstructive disease was well below $20 \%$ in the COPD and asthma groups in that study. This suggests that disease severity in each group tended to be low in this population-based study, and/or that some subjects were considerably underdiagnosed. On the other hand, both difficulty in differential diagnosis and lack of comprehension of these diseases were likely to contribute to a low diagnosis rate.

Some COPD patients have shown characteristics of asthma and have been diagnosed as asthmatics. Some asthma patients share the same features of COPD; yet, there has been no consensus on the definition and diagnostic criteria of overlap syndrome. According to the Dutch hypothesis, asthma, chronic bronchitis, and emphysema are different expressions of the same disease entity, and influenced by environmental and genetic influences. ${ }^{12,13} \mathrm{~A}$ cohort study has suggested that asthma could be a risk factor for COPD development. ${ }^{14}$

The clinical features of the overlap syndrome group in the current study were remarkable. The group showed a greater rate of history of structural diseases such as pulmonary tuberculosis and bronchiectasis than the other groups. This could be linked to lower socioeconomic status, since both low economic status and shorter education duration were associated with overlap syndrome. Additionally, $40 \%$ of subjects in the overlap syndrome group had been diagnosed with asthma in the past. These results could support the hypothesis that asthma and AHR predispose individuals to develop COPD or fixed airway obstruction later in life. ${ }^{12}$ Structural diseases can also be potential predisposing factors. ${ }^{15-18}$ Childhood respiratory infection has been associated with airway obstruction and/or bronchiectasis. ${ }^{19}$ Another explanation could be that overlap syndrome progressed under the influence of environmental factors like aging, smoking, or undertreatment due to low educational and socioeconomic status. These hypotheses could provide insights for further understanding of obstructive lung disease, including overlap syndrome.
Some degree of airflow obstruction of as much as $\mathrm{FEV}_{1}$ 25-30 mL/year is related to the natural aging process of lung function decrease. Airflow obstruction appears to be more of a fixed pattern in the elderly with asthma, whose symptoms worsen and respond poorly to treatment. ${ }^{20}$ Similarly, the prevalence of AHR in COPD patients is increased as much as $3 \times$ in the elderly compared to non-elderly patients. ${ }^{21}$ Studies have shown that $50 \%$ of the elderly with airway disease have airflow variability with some degree of irreversible airway obstruction., ${ }^{422}$ Likewise, aging and cigarette smoking are other strong risk factors for obstructive lung disease. Smoking increases neutrophilic inflammation and interferes with the anti-inflammatory actions of corticosteroids, ie, steroid resistance. ${ }^{23}$ Chronic inflammation and airway remodeling are influenced by smoking in both asthma and COPD patients.

Despite the fact that the COPD group had a greater number of subjects aged $\geq 60$ years with comorbidities and asthma group subjects had more preserved lung function, the COPD group had relatively fewer subjects with lower SRH compared to the asthma group. The overlap syndrome group showed the highest association with lower SRH, followed by the asthma group. The COPD group did not show any associations with the other groups, despite significant association between low $\mathrm{FEV}_{1}$ and lower $\mathrm{SRH}$. These results support the hypothesis that subjective symptoms rather than objective airflow obstruction results can affect individual SRH.

Many studies have focused on symptoms. The presence of dyspnea has been shown to predict 5 year survival better than airway obstruction in patients with COPD. ${ }^{24}$ The BODE Index is a better predictor of mortality than $\mathrm{FEV}_{1}$ alone; it includes BMI, airflow obstruction, the Modified Medical Research Council Dyspnea Scale (MMRC), and the 6 minute walk distance test for exercise capacity. ${ }^{25}$ The recent Global initiative for chronic Obstructive Lung Disease (GOLD) classification includes levels of daily symptoms, particularly self-reported dyspnea by MMRC scale, frequency of exacerbations, and level of lung function to stratify future risk. ${ }^{1}$ Using this classification, a population-based study demonstrated the role of dyspnea in COPD even among individuals with relatively preserved $\mathrm{FEV}_{1}{ }^{26}$

While a previous study showed that patients with overlap syndrome had lower health-related quality of life compared to those with asthma, ${ }^{27}$ the current study's asthma group rather than the COPD group showed lower SRH. This inconsistency could be due to differences in study subjects and the questionnaires used. Subjects were from a population-based survey, and most of them were more likely to have mild-to-moderate 
airflow limitation, whereas the previous study used patients with a diagnosis of asthma or COPD or both. ${ }^{27}$ In that study of search predictors of a decline in health-related quality of life in patients with asthma or COPD, respiratory symptoms were an important predictor of decline in health-related quality of life. ${ }^{28}$

The current study has several limitations. First, the examination survey was not solely designed for airway disease, and spirometry was not used in all participants. This resulted in selection bias. In addition, the overall participation rate was low, and only subjects with eligible spirometry results were enrolled. Subjects with no symptoms were excluded, and severely ill patients were excluded due to unsuitable data. Different sampling weights provided by KNHANES IV were used to minimize the healthy worker effect. ${ }^{8}$ Second, the presence of AHR was not determined based on objective methods but on a subjective questionnaire. This could have led to a misdiagnosis and heterogeneity of subjects in each group. Some asthmatics who were uncontrolled or who had underlying structural disease might have been misclassified to the overlap syndrome group. Some COPD patients with recent exacerbations could also have been misclassified. In the setting of a general population-based study however, the use of objective methods was unfeasible and impractical; although self-assessment methods have been widely used in previous studies. ${ }^{29,30}$ Third, there was no information about use of medications that could influence lung function such as inhaled corticosteroids and bronchodilators. Their potential use and effects were excluded, since pre-study omission was not implemented and questionnaires did not include regular medication use. However, most subjects were considered as not having been treated due to the low rate of physician diagnosis. Lastly, SRH is subjective and not a standardized item.

Health status is influenced by many other emotional and psychological factors. There were inter- and intra-individual differences in expression of SRH, even in the NOD group. Health status is not an appropriate marker to apply to all patients equally in practice. Nevertheless, a recent study reported that SRH was strongly associated with quality of life in asthma subjects. ${ }^{31} \mathrm{SRH}$ as a simple method for assessing health status related to disease has an advantage of being easier to explain to patients compared to a complicated respiratory questionnaire.

\section{Conclusion}

Obstructive lung disease is considered an aggregate of heterogeneous diseases with unique features. Compared to subjects with airway obstruction and no wheezing, subjects from our asthma and overlap syndrome groups showed lower SRH, although each subgroup did not represent a specific clinical disease entity. This study provides further evidence that overlap syndrome is an important and understudied disease, and that this population needs more appropriate care when considering the high burden of morbidity and lower SRH.

\section{Acknowledgments}

This research was supported by the Basic Science Research Program through the National Research Foundation of Korea (NRF) funded by the Ministry of Science, ICT \& Future Planning (2010-0027945).

\section{Author contributions}

Study concept and design, JH Lee; data acquisition and quality control, JW Chung, KA Kong, SJ Lee; analysis and interpretation: JW Chung, KA Kong, JH Lee; critical revision of manuscript, all authors. All authors contributed toward data analysis, drafting and revising the paper and agree to be accountable for all aspects of the work.

\section{Disclosure}

The authors report no conflicts of interest in this work.

\section{References}

1. Vestbo J, Hurd SS, Agusti AG, et al. Global strategy for the diagnosis, management, and prevention of chronic obstructive pulmonary disease: GOLD executive summary. Am J Respir Crit Care Med. 2013;187(4): 347-365.

2. Soriano JB, Davis KJ, Coleman B, Visick G, Mannino D, Pride NB. The proportional Venn diagram of obstructive lung disease: two approximations from the United States and the United Kingdom. Chest. 2003;124(2):474-481.

3. Hospers JJ, Postma DS, Rijcken B, Weiss ST, Schouten JP. Histamine airway hyper-responsiveness and mortality from chronic obstructive pulmonary disease: a cohort study. Lancet. 2000;356(9238):1313-1317.

4. Gibson PG, Simpson JL. The overlap syndrome of asthma and COPD: what are its features and how important is it? Thorax. 2009;64(8): 728-735.

5. Guerra S. Overlap of asthma and chronic obstructive pulmonary disease. Curr Opin Pulm Med. 2005;11(1):7-13.

6. Kim DS, Kim YS, Jung KS, et al. Prevalence of chronic obstructive pulmonary disease in Korea: a population-based spirometry survey. Am J Respir Crit Care Med. 2005;172(7):842-847.

7. Kim SY, Jung JY, Park MS, et al. Increased prevalence of self-reported asthma among Korean adults: an analysis of KNHANES I and IV data. Lung. 2013;191(3):281-288.

8. Yoo KH, Kim YS, Sheen SS, et al. Prevalence of chronic obstructive pulmonary disease in Korea: the fourth Korean National Health and Nutrition Examination Survey, 2008. Respirology. 2011;16(4): 659-665.

9. Freyer-Adam J, Gaertner B, Tobschall S, John U. Health risk factors and self-rated health among job-seekers. BMCPublic Health. 2011;11:659.

10. Executive Summary of The Third Report of The National Cholesterol Education Program (NCEP) Expert Panel on Detection, Evaluation, and Treatment of High Blood Cholesterol in Adults (Adult Treatment Panel III). JAMA. 2001;285(19):2486-2497. 
11. Pleasants RA, Ohar JA, Croft JB, et al. Chronic obstructive pulmonary disease and asthma-patient characteristics and health impairment. COPD. Epub October 23, 2013.

12. Orie NG. The dutch hypothesis. Chest. 2000;117(5 Suppl 1):299S.

13. Postma DS, Boezen HM. Rationale for the Dutch hypothesis. Allergy and airway hyperresponsiveness as genetic factors and their interaction with environment in the development of asthma and COPD. Chest. 2004;126(Suppl 2):96S-104S.

14. Silva GE, Sherrill DL, Guerra S, Barbee RA. Asthma as a risk factor for COPD in a longitudinal study. Chest. 2004;126(1):59-65.

15. Lee $\mathrm{CH}$, Lee MC, Lin $\mathrm{HH}$, et al. Pulmonary tuberculosis and delay in anti-tuberculous treatment are important risk factors for chronic obstructive pulmonary disease. PLoS One. 2012;7(5):e37978.

16. Edmond K, Scott S, Korczak V, et al. Long term sequelae from childhood pneumonia; systematic review and meta-analysis. PLoS One. 2012;7(2):e31239.

17. Hooper R, Burney P, Vollmer WM, et al. Risk factors for COPD spirometrically defined from the lower limit of normal in the BOLD project. Eur Respir J. 2012;39(6):1343-1353.

18. Lam KB, Jiang CQ, Jordan RE, et al. Prior TB, smoking, and airflow obstruction: a cross-sectional analysis of the Guangzhou Biobank Cohort Study. Chest. 2010;137(3):593-600.

19. Svanes C, Sunyer J, Plana E, et al. Early life origins of chronic obstructive pulmonary disease. Thorax. 2010;65(1):14-20.

20. Zeki AA, Schivo M, Chan A, Albertson TE, Louie S. The asthma-COPD overlap syndrome: a common clinical problem in the elderly. J Allergy (Cairo). 2011:861926.

21. Lindner K, Panaszek B, Machaj Z. [Asthma in the elderly]. Pol Arch Med Wewn. 2007;117(8):350-354. Polish.

22. Marsh SE, Travers J, Weatherall M, et al. Proportional classifications of COPD phenotypes. Thorax. 2008;63(9):761-767.
23. Chalmers GW, MacLeod KJ, Thomson L, Little SA, McSharry C, Thomson NC. Smoking and airway inflammation in patients with mild asthma. Chest. 2001;120(6):1917-1922.

24. Nishimura K, Izumi T, Tsukino M, Oga T. Dyspnea is a better predictor of 5-year survival than airway obstruction in patients with COPD. Chest. 2002;121(5):1434-1440.

25. Celli BR, Cote CG, Marin JM, et al. The body-mass index, airflow obstruction, dyspnea, and exercise capacity index in chronic obstructive pulmonary disease. N Engl J Med. 2004;350(10):1005-1012.

26. Lange P, Marott JL, Vestbo J, et al. Prediction of the clinical course of chronic obstructive pulmonary disease, using the new GOLD classification: a study of the general population. Am J Respir Crit Care Med. 2012;186(10):975-981.

27. Kauppi P, Kupiainen H, Lindqvist A, et al. Overlap syndrome of asthma and COPD predicts low quality of life. J Asthma. 2011;48(3): 279-285.

28. Hesselink AE, van der Windt DA, Penninx BW, et al. What predicts change in pulmonary function and quality of life in asthma or COPD? J Asthma. 2006;43(7):513-519.

29. Xuan W, Peat JK, Toelle BG, Marks GB, Berry G, Woolcock AJ. Lung function growth and its relation to airway hyperresponsiveness and recent wheeze. Results from a longitudinal population study. Am J Respir Crit Care Med. 2000;161(6):1820-1824.

30. ArifAA, Delclos GL, Lee ES, Tortolero SR, Whitehead LW. Prevalence and risk factors of asthma and wheezing among US adults: an analysis of the NHANES III data. Eur Respir J. 2003;21(5):827-833.

31. Syk J, Alving K, Unden AL. Association between self-rated health and asthma: a population-based study. Clin Respir J. 2012;6(3):150-158. 


\section{Supplementary material}

Table SI Comparison of characteristics between included and excluded subjects

\begin{tabular}{|c|c|c|c|c|c|}
\hline \multirow{3}{*}{$\begin{array}{l}\text { Variables } \\
\text { Males }\end{array}$} & \multirow{2}{*}{\multicolumn{2}{|c|}{$\frac{\text { Included }}{\mathrm{N}=9,104}$}} & \multirow{2}{*}{\multicolumn{2}{|c|}{$\begin{array}{l}\text { Excluded } \\
\mathrm{N}=9,302\end{array}$}} & \multirow{3}{*}{$\begin{array}{l}P \text {-value } \\
<0.0001\end{array}$} \\
\hline & & & & & \\
\hline & 4,067 & $44.7 \%$ & 3,857 & $41.5 \%$ & \\
\hline \multicolumn{6}{|l|}{ Age, years } \\
\hline $19-39$ & 2,410 & $26.5 \%$ & 3,845 & $41.3 \%$ & \multirow[t]{3}{*}{$<0.000$} \\
\hline $40-59$ & 4,062 & $44.6 \%$ & 2,603 & $28.0 \%$ & \\
\hline$\geq 60$ & 2,632 & $28.9 \%$ & 2,854 & $30.7 \%$ & \\
\hline \multicolumn{6}{|l|}{$\mathrm{BMI}, \mathrm{kg} / \mathrm{m}^{2}$} \\
\hline Missing & 0 & $0.0 \%$ & 1,213 & $13.0 \%$ & \multirow[t]{4}{*}{$<0.000$} \\
\hline$<8.5$ & 202 & $2.2 \%$ & 598 & $6.4 \%$ & \\
\hline $18.5-25$ & 5,518 & $60.6 \%$ & 5,431 & $58.4 \%$ & \\
\hline$\geq 25$ & 3,384 & $37.2 \%$ & 2,060 & $22.1 \%$ & \\
\hline \multicolumn{6}{|c|}{ Education duration, years } \\
\hline Missing & 0 & $0.0 \%$ & $\mathrm{I}, 159$ & $12.5 \%$ & \multirow[t]{5}{*}{$<0.000$ I } \\
\hline$\leq 6$ & 2,568 & $28.2 \%$ & 2,466 & $26.5 \%$ & \\
\hline $6-9$ & 1,196 & $13.1 \%$ & 730 & $7.8 \%$ & \\
\hline $9-12$ & 3,070 & $33.7 \%$ & 2,844 & $30.6 \%$ & \\
\hline$>12$ & 2,270 & $24.9 \%$ & 2,103 & $22.6 \%$ & \\
\hline \multicolumn{6}{|l|}{ Socioeconomic status } \\
\hline Missing & 0 & $0.0 \%$ & 546 & $5.9 \%$ & \multirow[t]{4}{*}{$<0.000$} \\
\hline$<25$ th percentile & 2,153 & $23.6 \%$ & 2,337 & $25.1 \%$ & \\
\hline $25-75$ th percentile & 4,597 & $50.5 \%$ & 4,295 & $46.2 \%$ & \\
\hline$>75$ th percentile & 2,354 & $25.9 \%$ & 2,124 & $22.8 \%$ & \\
\hline \multicolumn{6}{|l|}{ Smoking history } \\
\hline Missing & 0 & $0.0 \%$ & $\mathrm{I}, \mathrm{I} 58$ & $12.4 \%$ & \multirow[t]{4}{*}{$<0.0001$} \\
\hline Never-smoker & 5,327 & $58.5 \%$ & 5,195 & $55.8 \%$ & \\
\hline Ex-smoker & 1,696 & $18.6 \%$ & 1,268 & $13.6 \%$ & \\
\hline Current smoker & 2,081 & $22.9 \%$ & $|, 68|$ & $18.1 \%$ & \\
\hline \multicolumn{6}{|l|}{ Self-rated health } \\
\hline Missing & 0 & $0.0 \%$ & $\mathrm{I}, 152$ & $12.4 \%$ & \multirow[t]{3}{*}{$<0.0001$} \\
\hline Better & 6,957 & $76.4 \%$ & 6,035 & $64.9 \%$ & \\
\hline Lower & 2,147 & $23.6 \%$ & 2,115 & $22.7 \%$ & \\
\hline
\end{tabular}

Abbreviations: $\mathrm{N}$, number of subjects; BMI, body mass index.

\section{Publish your work in this journal}

The International Journal of COPD is an international, peer-reviewed journal of therapeutics and pharmacology focusing on concise rapid reporting of clinical studies and reviews in COPD. Special focus is given to the pathophysiological processes underlying the disease, intervention programs, patient focused education, and self management protocols.

\section{Dovepress}

This journal is indexed on PubMed Central, MedLine and CAS. The manuscript management system is completely online and includes a very quick and fair peer-review system, which is all easy to use. Visit http://www.dovepress.com/testimonials.php to read real quotes from published authors. 